\title{
EL USO FORMATIVO DEL PORTAFOLIOS DOCENTE EN EL ÁMBITO UNIVERSITARIO
}

\author{
Ángel Sobrino Morrás, Carlota Pérez Sancho, \\ Concepción Naval Durán
}

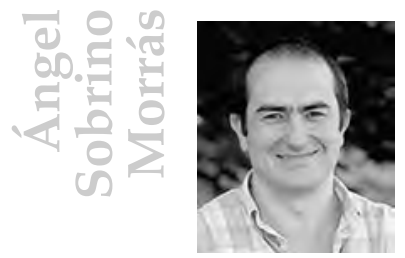

Doctor en Ciencias de la Educación, Universidad de Navarra. Licenciado en Psicología, UNED. Doctor de Tecnología Educativa, Universidad de Navarra. Director del Máster Universitario en Profesorado, Universidad de Navarra. Director del curso on line «TIC y buenas prácticas para la formación del profesorado». Destacan entre sus obras las coautorías de Integración curricular de las TIC, Madrid, Ariel, 2000; La formación en Internet, Madrid, Ariel, 2003. Correo electrónico: [asobrino@unav.es].

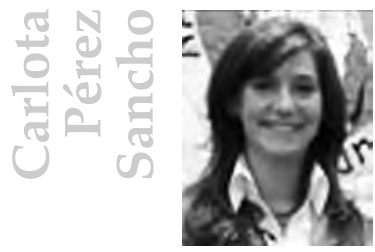

Doctora en Ciencias de la Educación, Universidad de Navarra. Su tesis se centró en el estudio del portafolios docente como instrumento formativo para el profesorado universitario. Desde 2006 hasta 2008 ha sido miembro del servicio de Innovación Educativa de la Universidad de Navarra, desarrollando programas de innovación educativa y desarrollo docente. Principales líneas de investigación: tecnología educativa; formación del profesorado; innovación educativa. Correo electrónico: [cperez1@alumni.unav.es].

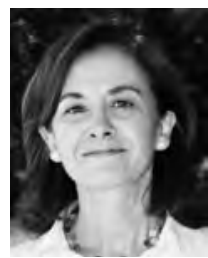

Doctora en Ciencias de la Educación. Becaria predoctoral del Programa de Formación de Personal Investigador del MEC y post-doctoral de la Fulbright Commission y de Caja Madrid. Actualmente es profesora titular de Teoría de la Educación y Vicerrectora en la Universidad de Navarra desde 2001. Dirige la revista Estudios sobre Educación y es miembro del Editorial Board del Journal of Social Science Education, y del Journal Citizenship, Teaching and Learning. Su investigación se centra en: la educación ciudadana y la participación social; el impacto social de las tecnologías de la comunicación en los jóvenes; la innovación educativa en la educación superior.

Correo electrónico: [cnaval@unav.es]. 


\title{
RESUMEN
}

El presente trabajo realiza una aproximación conceptual a la idea del portafolios docente y su empleo en el ámbito universitario. El concepto de portafolios docente encierra cierta ambigüedad debido a la amplia variedad de objetivos para los que puede usarse. Por ello es necesario delimitar de forma conceptual qué son los portafolios y los diversos tipos existentes. Una vez precisado a qué hace referencia el término "portafolios docente», se analizan las posibilidades de uso que brindan estos portafolios para el profesorado universitario. Por último, el trabajo se centra en establecer cuatro principios guía sobre los que conviene fundamentar el empleo eficaz del portafolios docente como instrumento formativo: la docencia como profesión, la reflexión y los procesos metacognitivos, la evaluación como instrumento de mejora, y los sistemas de apoyo y seguimiento como la tutoría o el mentorazgo.

Palabras clave: universidad; portafolios docente; formación del profesorado.

\begin{abstract}
This article describes a conceptual approach to the concept of the teaching portfolio and its use in higher education. The concept of the teaching portfolio involves a certain ambiguity, due to the wide variety of objectives for which it can be used. Therefore, it is necessary to make a conceptual delimitation of the teaching portfolio, as well as the diverse types which exist. Once the reference of the term has been defined, the article studies the possibilities of use that these teaching portfolios offer the faculty. Finally, the article establishes four principles to guide the effective use of these portfolios as a formative instrument: the profession of teaching, reflection and processes of metacognition, evaluation as tool for teaching improvement, and support systems such as mentoring.
\end{abstract}

Keywords: higher education; teaching portfolio; training faculty. 


\section{INTRODUCCIÓN}

El presente trabajo realiza una aproximación conceptual a la idea del portafolios docente y su aprovechamiento en el ámbito universitario. Ello encierra cierta ambigüedad debido a la amplia variedad de objetivos para los que puede utilizarse. Por esto es necesario delimitar de forma conceptual qué son los portafolios y los diversos tipos existentes. Una vez precisado a qué hace referencia el término "portafolios docente», se analizan sus posibilidades de uso para el profesorado universitario. Por último, el trabajo se centra en establecer cuatro principios guía sobre los que conviene fundamentar el manejo eficaz del portafolios docente como instrumento formativo: la docencia como profesión, la reflexión y los procesos metacognitivos, la evaluación como instrumento de mejora, y los sistemas de apoyo y seguimiento como la tutoría o el mentorazgo. El mayor interés está en conocer dichos principios para estar en mejor disposición de aplicar, de forma efectiva, los portafolios docentes como instrumento de formación del profesorado.

\section{II. ¿QUÉ ES EL PORTAFOLIOS Y QUÉ TIPOS EXISTEN?}

La idea del uso de portafolios viene prestada de otros ámbitos profesionales. Parece que nace en el mundo del arte y de la arquitectura, donde los profesionales reunían en una carpeta -o portafolios ${ }^{1}$ - sus mejores obras, proyectos o trabajos a modo de currículum vítae o carta de presentación (Seldin, 1991; Lyons, 1998; Agra, Gewerc y Montero, 2002; Tucker, Stronge y otros, 2002; Fernández March, 2004).

Posteriormente, la concepción del portafolios como «colección intencionada de trabajo para su análisis y reflexión» (Tucker, Stronge y otros, 2002, 2) o «espejo profesional» (Martin-Kniep, 1999, 17) fue introducida hace ya más de una década en el ámbito educativo, aunque de modos diversos y con distintos propósitos (Bird, 1990; Edgerton, Hutchings y Quinlan, 1991; Kingore, 1993).

1 El Diccionario de la Lengua Española de la Real Academia Española (RAE) define portafolio o portafolios como: «Cartera de mano para llevar libros, papeles, etcétera». 
Las características definitorias del portafolios han sido analizadas por diversos autores y pueden reducirse a tres: se trata de un documento personal, por lo que habrá tantos portafolios como personas los aprovechen; acumula información sobre el desarrollo de la persona que lo realiza; y proporciona una descripción objetiva, y por lo tanto los datos y opiniones vertidas en él deben archivarse de forma sistemática y debidamente contrastados, acreditando de alguna manera lo que se afirma.

Según Smith y Tillema (2003), el término portafolios se ha convertido en un vocablo popular, una "palabra de moda» (buzzword), sin quedar siempre claro qué significa o implica el término dada la amplia variedad de objetivos para los que puede emplearse. Estos autores advierten del peligro y las tensiones que pueden surgir en el uso del portafolios, ya que cada uno de ellos requiere un diverso proceso de recolección y selección de evidencias y, por lo tanto, su resultado sirve para propósitos distintos.

Conviene distinguir los diferentes tipos de portafolios con el propósito de clarificar y especificar a qué hace referencia ese término. Para ello, pueden realizarse diversas clasificaciones atendiendo a distintos aspectos. Smith y Tillema $(2001,2003)$ señalan dos dimensiones: a) el propósito del portafolios, orientado a la selección o promoción, o al aprendizaje o el desarrollo; b) el marco en el que se usa, exigido por requisitos externos, o iniciado y dirigido de forma voluntaria y para su empleo personal. Estas dos dimensiones originan cuatro tipos de portafolios: el dossier (dossier portfolio) es un registro de logros, exigido por requisitos externos con fines de selección o promoción; el portafolio de formación (training portfolio), requerido por circunstancias externas, su importancia radica en el producto final y se orienta hacia una evaluación externa como en el caso anterior, pero de naturaleza más formativa; el portafolios reflexivo (reflexive portfolio) es una recopilación de trabajo útil y personal que muestra el crecimiento y los logros adquiridos por una persona, con fines de promoción o admisión y con propósito formativo; y el portafolios para el desarrollo personal (personal development portfolio) se basa en la evaluación personal y el registro reflexivo del propio crecimiento profesional a largo plazo. 
A continuación se propone, de manera esquemática, otra posible agrupación de los portafolios, basada en tres categorías: según su usuario, su finalidad o empleo, y su formato. Ello da lugar a seis tipos de portafolios:

Tabla 1. Clasificación de los tipos de portafolios.

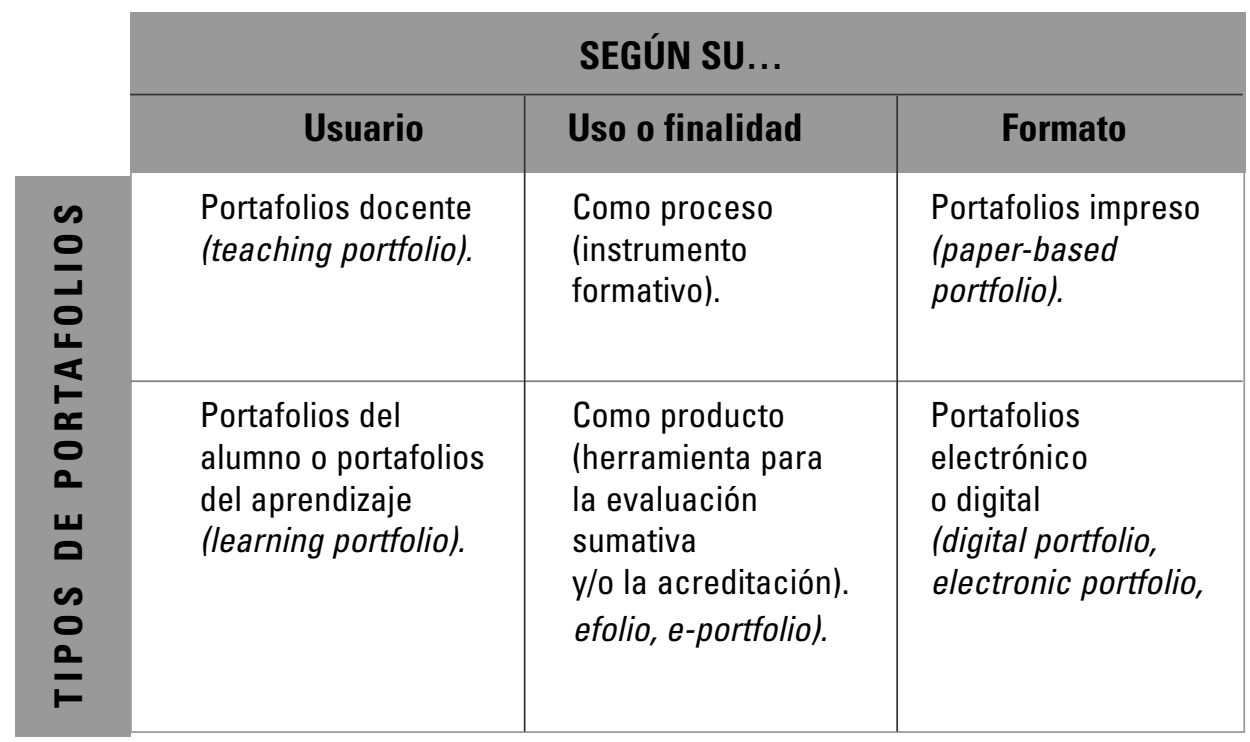

Esta clasificación -fruto del estudio teórico del tema- es análoga a la propuesta por Smith y Tillema $(2001,2003)$ pero aporta, junto al propósito de los portafolios, otras dos dimensiones relativas al usuario y a su formato, dando lugar a seis tipos de portafolios. Éstos pueden, a su vez, combinarse entre sí resultando otras modalidades: pueden existir portafolios docentes en formato impreso con fines formativos, portafolios del alumno en formato digital con fines evaluadores, etcétera. A continuación se detalla brevemente en qué consiste cada uno de estos seis tipos principales de portafolios. 


\section{CLASIFICACIÓN SEGÚN SU USUARIO}

\section{- Portafolios del estudiante}

También denominado portafolios de aprendizaje (learning portfolio), se utiliza como herramienta didáctica evaluadora del proceso de aprendizaje del alumno. En este tipo de portafolios, el alumno recoge los diversos trabajos académicos elaborados en un período de tiempo determinado -trimestre, semestre, curso, etcétera-, en una asignatura o programa concreto, acompañados de su propia reflexión acerca de aspectos tales como el esfuerzo que ha supuesto elaborarlo, el proceso de aprendizaje que ha seguido o las principales dificultades con las que se ha encontrado, entre otros.

Se trata del portafolios de formación al que hacen referencia Smith y Tillema (2001, 2003), y que ha sido muy estudiado en los últimos años por diversos autores (cf. Kingore, 1993; Klenowski, 2002; Smith y Tillema, 2003; Zubizarreta, 2004; Cano, 2005; Whittier y Lara, 2005).

El uso de este tipo de portafolios con fines evaluativos aporta una nueva perspectiva sobre el aprendizaje al documentar el proceso de aprendizaje del alumno y evaluar con una variedad de evidencias cómo los objetivos educativos han sido logrados. Por todo ello, representa una alternativa ante la cada día más creciente insatisfacción con la evaluación sólo cuantitativa. El portafolios se presenta como un método valioso que apoya una visión del aprendizaje del alumno basada en la experiencia y descubrimiento de significado, desarrolla la adquisición de conocimientos y favorece el proceso de reflexión del alumno (Wade y Yarbrough, 1996).

Klenowski (2000) establece una serie de principios que sustenta el empleo de este tipo de portafolios con fines evaluativos: proporciona una nueva perspectiva sobre el aprendizaje, el alumno se involucra activamente en el proceso, y se promueve una sana y equilibrada independencia en el aprendizaje. Es un instrumento de mejora en un período de tiempo determinado; su fortaleza 
reside en el propio proceso que documenta los logros y analiza las experiencias de enseñanza-aprendizaje; la autoevaluación es parte integrante del desarrollo del portafolios; y potencia la capacidad de recoger, seleccionar y reflejar el trabajo realizado.

\section{- Portafolios del docente}

También conocido como «carpeta docente» (teaching portfolio, teacher portfolio o professional portfolio), lo emplea el profesor para recoger evidencias de su labor diaria, con el propósito de convertirse en un instrumento que facilite la reflexión sobre su propia práctica -medio para su desarrollo profesional- y también como instrumento de evaluación -o autoevaluación- de su labor.

Aunque en el ámbito nacional parece que el concepto de este tipo de portafolios es algo nuevo o innovador, su aplicación es una realidad desde hace ya mucho tiempo. Este concepto de portafolios fue introducido por la Asociación Canadiense de Profesores de Universidad en 1980 y desde entonces ha sido adoptado en universidades de todo el mundo (Knapper y Wilcox, 1998, 5).

Hace ya más de diez años, Seldin $(1991,3)$ definía el portafolios docente como:

Una descripción objetiva de las principales fortalezas y logros de un profesor en su práctica docente. Recoge documentos y materiales que, de modo conjunto, dan indicios del alcance y la calidad del ejercicio docente de un profesor. Significa para la enseñanza lo que las listas de publicaciones, becas y premios son para la investigación.

Este tipo de portafolios suele recoger el quehacer de un docente en su conjunto, pero también existe una modalidad que se centra exclusivamente en un curso o asignatura específica (course portfolio). En estos casos, su interés radica en su utilidad para mejorar la instrucción y el aprendizaje en una clase o asignatura concreta (Cerbin, 1994; Zubizarreta, 1995; Hutchings, 1998). 


\section{CLASIFICACIÓN SEGÚN SU USO O FINALIDAD}

Se ha señalado ya que las posibilidades de aplicación del portafolios con fines educativos son muy diversas. Klenowski (2002, 22-37) diferencia cinco posibilidades de uso del portafolios: en la evaluación sumativa; como base para la certificación de competencias y la selección de candidatos; con fines valorativos y de promoción; como apoyo para la enseñanza y el aprendizaje; y en el desarrollo profesional.

Sin embargo, al hacer referencia a los tipos de portafolios según su uso o finalidad, a partir de la clasificación presentada en la Tabla 1, se distinguen sólo dos tipos de portafolios: como producto o como proceso. La razón es que se considera que estas dos posibilidades engloban al resto, comprendiendo dentro del portafolios como producto todos aquellos portafolios cuyo propósito es «rendir cuentas»-que englobarían los tres primeros tipos que cita Klenowski- y, como proceso, los que persiguen el desarrollo y la mejora personal -los dos últimos señalados por este autor-. Además, esta clasificación en dos tipos de aplicaciones concuerda con la aportada por Smith y Tillema (2001, 2003), quienes diferenciaban el portafolios orientado a la selección o promoción (entendido como producto) y el orientado al aprendizaje o el desarrollo (entendido como proceso). Estos dos tipos de portafolios serán tratados con más detenimiento en el siguiente epígrafe al abordar estas dos posibilidades de empleo específicas de los portafolios docentes. Sin embargo, se definen ahora brevemente y de manera general.

\section{- El portafolios como proceso}

El portafolios se considera un instrumento formativo cuando su finalidad es tal, basada en el propio proceso reflexivo y de autoevaluación que tiene lugar durante la creación del portafolios. Su importancia radica en el proceso de desarrollo del portafolios en sí mismo y no tanto en su resultado final. 


\section{- El portafolios como producto}

Se considera como herramienta evaluadora cuando su finalidad es la evaluación sumativa o acreditación, basada en las evidencias que muestran la competencia del autor del portafolios. Mientras en el caso anterior lo importante era el proceso, la evolución, progreso y desarrollo del portafolios, en éste, el interés se centra en el resultado o producto final, el portafolios una vez elaborado.

\section{CLASIFICACIÓN SEGÚN SU FORMATO}

Por último, teniendo en cuenta el formato, el portafolios puede realizarse de forma impresa o digital.

\section{- Portafolios impreso}

Se trata del formato que tenían los primeros portafolios que comenzaron a crearse; muchos continúan realizándose en papel (paper-based portfolio). Los diversos documentos y papeles se almacenan en carpetas o archivadores que posteriormente se muestran, habitualmente de forma personal, a la persona interesada. Algunas publicaciones que versan sobre el portafolios docente recogen ejemplos reales y concretos de este tipo de portafolios (Edgerton, Hutchings y otros, 1991; Seldin, 1991, 1993, 2004; Knapper y Wilcox, 1998; Campbell, Cignetti y otros, 2004).

\section{- Portafolios digital}

Se designa con diversos términos: digital portfolio, electronic portfolio, efolio, e-portfolio, webfolio, web-based electronic portfolio. Todos ellos aluden a los portafolios realizados de manera electrónica o con un formato basado en el uso del ordenador ${ }^{2}$.

2 Una muestra de la creciente utilización del portafolios en formato digital por parte de las instituciones se encuentra en el listado realizado por la AAHE (American Association for Higher Education) en colaboración con el centro de enseñanza-aprendizaje de la Universidad de Denver. En: [http://ctl.stanford.edu/Tomprof/postings/762.html]. Consultado: 9-XII-2008. 
El hecho de aprovechar las herramientas tecnológicas como recipientes de los portafolios no modifica lo que son o pueden llegar a ser. Sin embargo, se benefician de una serie de ventajas en comparación con el formato impreso, relacionadas con la accesibilidad que les proporciona ser publicados en la web y la flexibilidad que comporta este tipo de formato para la organización del contenido. En este tipo de portafolios puede incorporarse video, audio, ilustraciones o fotografías, texto, presentaciones de diapositivas, $\mathrm{u}$ otros recursos. Esta modalidad permite aportar evidencias que en formato papel no suelen incluirse como, por ejemplo, los diversos recursos tecnológicos empleados en las clases. Se trata de una manera más sencilla y atractiva de presentar el material, de forma que su visualización sea más fácil y ordenada.

Campbell, Cignetti, Melenyzer, Nettles y Wyman (2004, 20-21) señalan cuatro beneficios derivados de la utilización de portafolios en formato electrónico: demuestra los conocimientos y las habilidades tecnológicas que posee la persona que lo desarrolla; facilita su distribución; almacena muchos documentos; y aumenta su accesibilidad.

Barrett (2005a, 2005b) ha experimentado y estudiado con detalle los diferentes tipos de soportes o programas existentes para desarrollar portafolios on line. Las herramientas disponibles para realizar estos portafolios son numerosas; analizar de forma concreta cada una de ellas excede los límites del presente artículo ${ }^{3}$. Sin embargo, conviene recoger la clasificación realizada por Lorenzo e Ittelson (2005), los cuales compendian las posibilidades disponibles, distinguiendo cuatro procedimientos que pueden emplear las instituciones para desarrollar portafolios digitales:

1. Desarrollo de software específico en la propia institución. Los informáticos de la institución pueden desarrollar de manera local su propio sistema. De esta manera: se desarrolla justamente aquello que

3 Por ello, para un estudio más detallado se remite al trabajo desarrollado por esta autora. BARRETT, H.C. (2005a). My «On line Portfolio Adventure» (Versions of my on line portfolios developed using different systems or on line publishing tools). En: [http:/ / electronicportfolios.org/myportfolio/versions.html]. Consultado: 9-XII-2008. 
se requiere; no hay necesidad de obtener licencias de uso; los derechos de propiedad intelectual son de la propia institución. Sin embargo, las posibles limitaciones de esta opción son que los costos del desarrollo de software y hardware pueden ser prohibitivos; el sistema necesita mucho más tiempo y energías en su desarrollo; se precisa un alto nivel de experiencia técnica sobre el desarrollo de software para construir y mantener el sistema; la institución puede no ser capaz de conservar a los empleados para que mantengan el sistema.

2. Utilización de software de código abierto. Son aquellos programas disponibles de forma pública sin necesidad de pagar por ellos. Las ventajas son obvias al no requerir pago alguno. Sin embargo, su mantenimiento y soporte técnico sí que supone costos asociados y pueden no cubrir las necesidades específicas de una institución, si bien es cierto que al ser de código abierto puede modificarse -parcialmente- de forma local para adaptarse a las necesidades concretas.

Lorenzo e Ittelson (2005) señalan, como ejemplo, Open Source Portfolio Initiative (OSPI). Este programa, analizado también por Barret (2005a), es fruto de un proyecto de colaboración entre diversas organizaciones e instituciones que persiguen el desarrollo de software de código abierto para el desarrollo de portafolios digitales en la educación. El proyecto fue constituido en enero de 2003 por la Universidad de Minnesota, la Universidad de Delaware, y rSmart Group ${ }^{4}$; la primera versión de Open Source Portfolio (OSP) fue desarrollada en julio de 2003, basada en el software para portafolios electrónicos de la Universidad de Minnesota. Las prestigiosas organizaciones e instituciones implicadas en el proyecto ${ }^{5}$ avalan de alguna manera su calidad

4 El grupo rSmart se dedica a fomentar el desarrollo de software de código abierto en educación. En: [http:/ / www.rsmart.com/]. Consultado: 9-XII-2008.

5 Entre las instituciones y organizaciones que desempeñan cargos directivos dentro de OSPI se señalan: la Universidad de Minnesota, Delaware, Rhode Island, Michigan, Indiana, la Carnegie Foundation for the Advancement of Teaching y el grupo rSmart. Otras insituciones que contribuyen en la iniciativa son: California State University System, Iowa State University, Georgetown University, Virginia Tech, Central Florida University, Clayton College, Framingham, University of Buffalo. Además OSPI esta relacionado con la Open Knowledge Initiative (OKI) y el IMS Global Learning Consortium. 
y hacen confiar en las posibilidades de OSP, su gran potencial a largo plazo y la garantía de un rápido y constante desarrollo de la herramienta ${ }^{6}$.

3. Empleo de software comercial. Es el software desarrollado por empresas con fines lucrativos. No exige gastos de desarrollo del software, el soporte técnico lo gestiona el vendedor y existen numerosos sistemas entre los cuales escoger. Sin embargo, las licencias se adaptan a los precios del vendedor y pueden incrementarse sin previo aviso, el servicio de atención al cliente y el soporte técnico pueden ser limitados. Un ejemplo de este tipo de software es Folio ${ }^{7}$.

4. Aplicación de herramientas de uso común. No es necesario contar con programas muy complejos para elaborar un portafolios en formato digital, a veces basta utilizar de modo adecuado herramientas que están al alcance de cualquier usuario hoy en día, como un procesador de textos, una base de datos o un programa de presentaciones en diapositivas ${ }^{8}$.

\section{POSIBILIDADES DE USO DEL PORTAFOLIOS DOCENTE}

En el epígrafe anterior se distinguían dos tipos de portafolios atendiendo a su finalidad: el empleado como herramienta para la evaluación sumativa o acreditación -entendido como producto- y el empleado como instrumento formativo -entendido como proceso-. A continuación se concreta su estudio en el caso de los portafolios docentes.

6 Crecimiento y desarrollo que se evidencia en las distintas versiones que han sido desarrolladas de la misma herramienta.

7 Folio ${ }^{\mathrm{TM}}$ by Eportato. En: [http:/ / eportaro.com/index.html]. Consultado: 9-XII-2008.

8 Para mayor información sobre cómo desarrollar un portafolios electrónico con herramientas o software de uso común, puede consultarse: Barrett, H.C. (2003), «At-a-Glance Guides» for Electronic Portfolio Development using common software tools. En: [http: / / electronicportfolios.org/ ALI/ataglance.pdf]. Consultado: 9-XII-2008. 


\section{EL PORTAFOLIOS DOCENTE COMO}

PRODUCTO: HERRAMIENTA PARA LA

\section{EVALUACIÓN SUMATIVA O LA ACREDITACIÓN}

Aunque el presente artículo pretende centrarse en el empleo del portafolios docente como instrumento formativo y de desarrollo profesional, no puede obviarse que se trata también de un recurso eficaz de evaluación sumativa y, de hecho, es más común su aplicación como herramienta de acreditación que de formación propiamente dicha.

Desde el punto de vista de su misión certificadora, cobra mayor sentido la acumulación de evidencias cuyo propósito es demostrar la valía profesional de un determinado profesor. Esta aplicación del portafolios, considerado como una herramienta de evaluación sumativa, puede ser utilizado con diversos objetivos como, por ejemplo, la obtención de premios y subvenciones relacionadas con la docencia, evaluar a los candidatos a nuevas plazas, o como criterio de evaluación de cara a la promoción del profesorado.

El portafolios puede resultar un instrumento muy eficaz al servicio de la evaluación del docente, mostrando algunas ventajas con respecto a otros métodos de valoración que puedan ser utilizados. Seldin $(2004,3)$ lo compara con técnicas de evaluación como las efectuadas por parte de los alumnos, o las observaciones de los pares comparando a éstas con «flashes» que iluminan tan sólo -de modo instantáneo, en un momento determinado- las habilidades y aptitudes docentes, frente al portafolios, que califica de «reflector», capaz de mostrar una amplia gama de habilidades, aptitudes, actitudes y valores del profesor. Una de las virtudes del portafolios es que posibilita una evaluación a través del seguimiento y documentación durante un período continuado en el tiempo, mayor que el permitido, por ejemplo, por las observaciones de los pares (Shulman, 1998). En palabras de Klenowski $(2004,60)$ :

El portafolios es una forma «expansionista» de evaluación, ya que permite la incorporación de múltiples fuentes y una variedad de medidas de evaluación. 
No puede olvidarse que cada tipo de portafolios requerirá un procedimiento de evaluación que se adecue a su objetivo particular. Es conveniente por ello que previamente se clarifiquen los criterios y estándares con los que se calificará cada portafolios. A este respecto, algunos autores han realizado listados con algunos criterios de evaluación de un portafolios docente (Feixas y Valero, 2003; Zubizarreta, 2004).

\section{EL PORTAFOLIOS DOCENTE COMO PROCESO: INTRUMENTO PARA LA FORMACIÓN DEL PROFESORADO}

La clasificación que se realiza de los portafolios, con fines certificativos (evaluación) o como medio para el desarrollo profesional (formativos), se trata tan sólo de un recurso teórico -utilizado por la mayoría de los especialistas- para diferenciar de modo adecuado sus dos posibles finalidades. Sin embargo, en muchas ocasiones, dicha distinción no es posible realizarla en la realidad.

Como afirma Tillema $(1998,263)$, el portafolios con fines acreditativos refleja tres conceptos principales sobre la evaluación que lo hace un método valioso para el desarrollo profesional y la formación: ayuda a la persona a supervisar su propio desarrollo, promoviendo por ello reflexión desde la práctica; establece o destaca las diferencias entre las percepciones de uno mismo y las opiniones externas sobre su comportamiento; es la propia persona la que posee las pautas y es destinataria de los beneficios de la información recogida.

Por eso, incluso en los casos en que el portafolios se emplea con fines de acreditación, posee una función formativa explícitamente innegable, aunque no haya sido perseguida.

Los portafolios, entendidos como instrumentos al servicio de la formación y el perfeccionamiento, se convierten en una herramienta útil para el desarrollo profesional (Seldin, 1991; 
Klenowski, 2000; Smith y Tillema, 2001; Stanley, 2001; Cano, 2003; Feixas y Valero, 2003; Fernández March, 2003, 2004).

Se ha comprobado que este uso del portafolios -concebido como proceso-, destinado a la autoevaluación, la formación, el mentorazgo y la evaluación formativa, es más común en Europa, mientras que el empleo anterior, como producto acabado orientado hacia la evaluación sumativa o acreditación, es más frecuente en Estados Unidos (Whittier y Lara, 2005). El propio contexto -evaluativo o formativo- en el que los portafolios son creados, afecta al modo de leerlos o revisarlos, y no debe ser el mismo en uno $\mathrm{u}$ otro caso (Conrad y Bowie, 2006).

La aplicación del portafolios como instrumento formativo es muy frecuente en profesionales de la medicina y la enfermería como medio para facilitar el crecimiento profesional, aprendiendo desde la práctica diaria en el lugar de trabajo (Clay, Petrusa, Harker y Andolsek, 2007; Duque, Finkelstein, Roberts, Tabatabai, Gold y Winer, 2006; Holmboe, Rodak, Mills, McFarlane y Schultz, 2006; Wood y May, 2006). Y se muestra, del mismo modo, como un instrumento formativo eficaz en el campo de la formación del profesorado y su desarrollo profesional continuo (Barrett, 2000, 2005c; Constantino y de Lorenzo, 2002; Klenowski, Askew y Carnell, 2006; Wray, 2007).

Según Klenowski (2004) entre los resultados pretendidos con el aprovechamiento de los portafolios en la formación del profesorado, se incluyen los siguientes (p. 53): desarrollo del pensamiento reflexivo; mayor conciencia de los estilos de enseñanza y aprendizaje; desarrollo de los valores propios y de la filosofía educativa; ampliación del conocimiento; incremento de la profesionalidad; conciencia de altos estándares y de estándares profesionales; autoevaluación con fines de mejora; promoción del aprendizaje de los estudiantes; provisión de medios de aprendizaje estimulantes, y utilización de un aprendizaje y evaluaciones auténticos.

En este contexto conviene conocer que un estudio realizado por Smith y Tillema (2001, 199-200) sobre las influencias a largo plazo del 
portafolios en el desarrollo profesional, revela que su uso de forma voluntaria -fruto de la iniciativa personal- promueve la autoevaluación y conduce a un mayor desarrollo profesional, en comparación con los portafolios realizados por requerimientos externos.

Además, una ventaja que ofrece el portafolios en la formación del profesorado es que se trata de una herramienta eminentemente contextual, es decir, capaz de ayudar al profesorado a mejorar de manera eficaz su práctica educativa desde su propia práctica.

Por último, reseñan también que el empleo de portafolios como herramienta de formación redunda en beneficio para el departamento o la propia institución en su conjunto (Ginés, Davis y otros, 2005). De esta manera los profesores con más experiencia podrían compartir, a través de sus portafolios, sus logros con los menos experimentados, pueden ser fuentes de inspiración e ideas para la creación de nuevas asignaturas, o el legado que un profesor puede aportar al departamento al retirarse.

\section{PRINCIPIOS EN LOS QUE SE BASA EL USO FORMATIVO DEL PORTAFOLIOS DOCENTE}

La utilización del portafolios docente como instrumento formativo se fundamenta en una serie de principios que lo contemplan como herramienta al servicio del desarrollo profesional. El interés del presente trabajo radica en conocer y describir dichos principios para estar en mejor disposición de aplicar, de forma efectiva, los portafolios docentes como instrumento de formación y mejora del profesorado.

Dichos principios han sido señalados, de uno u otro modo, por los diversos estudiosos (Freidus, 1998; Lyons, 1998; Tillema, 1998; Martin-Kniep, 1999; Cano, 2003, 2005; Klenowski, 2004), y pueden sintetizarse en cuatro: la docencia como profesión; la reflexión; la evaluación como instrumento de mejora; y los sistemas de apoyo o mentorazgo. A continuación se estudia de manera detallada cada uno. 


\section{LA DOCENCIA COMO PROFESIÓN}

Las nuevas demandas que el proceso de convergencia europea y la sociedad actual plantean a la Universidad, reclaman con más fuerza la profesionalización de la tarea docente. Hablar de profesionalización docente supone identificar una serie de parámetros que definan la profesión docente, que servirán de referencia para el diseño de unas políticas de formación pedagógica coherentes para el profesorado universitario.

El empleo del portafolios como herramienta formativa cuenta con la existencia de dichos parámetros de las competencias docentes del profesorado, y su desarrollo consiste en recoger información o evidencias procedentes de la labor diaria del profesor que corroboren o acrediten la consecución de dichos parámetros del profesional docente. De esta manera, el portafolios resulta un «espejo de la competencia» docente del profesor, que le aporta la retroalimentación necesaria sobre sus fortalezas y debilidades en el ejercicio diario de su profesión. Representa una alternativa de formación pedagógica para los profesores y les proporciona las herramientas conceptuales y prácticas que les permiten transformar su praxis.

El propio proceso de desarrollo de un portafolios -aunque pueda resultar una tarea ardua-favorece que el profesor adquiera una visión más cabal sobre sus capacidades y que establezca con mayor perspectiva los itinerarios de su futuro desarrollo (Smith y Tillema, 2001). En este contexto el portafolios se define como «un tipo de credencial representativa del nuevo profesionalismo docente» (Lyons, 1998, 19).

El uso del portafolios ayuda a darle mayor peso a la tarea docente a la hora de evaluar al profesor, que también realiza otro tipo de tareas, tales como la investigación o la gestión, cuyos resultados a menudo son más fáciles de mostrar que los docentes. Por ello, puede afirmarse que el portafolios contribuye a promover una cultura de profesionalismo en la docencia y de reconocimiento por sí misma (Crispín y Caudillo, 1998). 


\section{LA REFLEXIÓN}

\section{Y LOS PROCESOS METACOGNITIVOS}

De entre todas las características que definen a un buen docente, la capacidad de reflexionar sobre la propia enseñanza es una de las más importantes y, por esta razón, es necesario introducir estrategias formativas que permitan su desarrollo (Fernández March, 2004).

Como advierte Lyons (1998, 145):

Sin la capacidad de reflexionar, de poder pensar e interrogarse sobre su práctica, cuestionar su propio aprendizaje y el de sus alumnos, los docentes nunca querrían o podrían dejar de lado su rol de relatores y unirse a sus alumnos como copartícipes en la construcción del conocimiento.

La aplicación del portafolios se revela como una manera efectiva de mejorar la capacidad reflexiva necesaria para favorecer el desarrollo profesional del profesorado (Klenowski, 2000). El interés del portafolios como instrumento formativo no consiste en mostrar una imagen positiva del profesor capaz de presentar ante terceras personas su competencia profesional -ésa sería una visión certificativa del portafolios, entendido como producto acabado-. El potencial del portafolios en este sentido radica en que permite aportar un retrato veraz y sincero de dónde se encuentra el profesor dentro de su proceso de desarrollo profesional (Campbell, Cignetti y otros, 2004, 53). Esto es así hasta tal punto que Barrett y Carney (2003) afirman que: «La reflexión es el corazón y el alma del portafolios».

La clave del uso del portafolios como instrumento de mejora de la calidad de la docencia está en la reflexión del profesorado y en su consideración como un proceso abierto. Tras una primera valoración sobre los aspectos en los que se necesita mejorar, pueden formularse objetivos a corto o medio plazo que, una vez logrados, pasarán a formar parte de las nuevas evidencias a introducir en el portafolios; este proceso se repite de forma cíclica a lo largo de la carrera docente del profesor. 
Es decir, la elaboración de un portafolios supone un proceso en constante evolución en el que habrá que ir incluyendo nuevos materiales o sustrayendo aquellos que ya no interesan. Según Martin-Kniep (1999, 43):

La utilidad de los portafolios profesionales aumenta si los docentes los encaran como documentos vivos y crecientes del aprendizaje, en lugar de como receptáculos pasivos y estáticos de su trabajo.

De esta manera, la reflexión que se produce con el proceso de desarrollo del portafolios tiene lugar sobre lo reflejado en el portafolios, las prácticas docentes pasadas, pero también a través de nuevas metas o propósitos que se fijan de cara al futuro (cf. Lyons, 1998).

Se ha visto que cuando los profesores trabajan para desarrollar su portafolios docente, «llegan a saber más sobre sí mismos, sobre sus ideas personales y profesionales que impulsan su práctica» (Freidus, 1998, 94). El portafolios le brinda al sujeto el control sobre su propio aprendizaje, haciendo que identifique sus fortalezas y debilidades cuando éstas están en pleno proceso de desarrollo (Tillema y Smith, 2000). De esta manera, favorece la aparición de procesos metacognitivos en los profesores, ya que a través del portafolios, son capaces de reflexionar sobre sus propias ideas, su propio pensamiento y sus conocimientos, así como sobre su itinerario de aprendizaje durante el transcurso del tiempo.

En palabras de Freidus $(1998,81)$ :

La teoría que sustenta a los portafolios concuerda con la idea básica de Dewey de que el aprendizaje comprende un continuo de experiencias en el que un conocimiento se construye sobre conocimientos y valores previos, y es mediado por éstos. En los portafolios, la reflexión consciente sobre las propias experiencias pedagógicas sirve como un vehículo para el desarrollo profesional. 


\section{LA EVALUACIÓN COMO INSTRUMENTO DE MEJORA}

En el contexto del portafolios, la evaluación puede ser definida como:

[...] un proceso de estimación de la calidad de la enseñanza, asentado en evidencias y realizado para poder comprender y mejorar la práctica educativa (Santos, 1995, 88).

Es decir, la evaluación entendida como instrumento al servicio de la mejora docente, con miras al desarrollo profesional del sujeto. Se trata de una evaluación formativa, donde lo que prima es el propio proceso y no los resultados, enfocada al perfeccionamiento que proporciona al profesor los datos necesarios para comprender los procesos de enseñanza-aprendizaje y retroalimentar su práctica docente (Santos, 1995; Vera, 2001).

El concepto de evaluación es entendido como herramienta para la mejora:

Desde esta perspectiva, la finalidad primordial de todo proceso evaluativo es su función formativa en tanto que posibilita al profesorado tomar conciencia sobre sus fortalezas y debilidades profesionales, y planificar estrategias que incidan en la mejora de su actividad docente y su desarrollo como profesional (De Miguel, 1998, 70).

En palabras de Santos (1995, 29): «La autoevalución es un proceso de autocrítica que genera unos hábitos enriquecedores de reflexión sobre la propia realidad». La autoevaluación permite que sean los mismos profesores los autores del cambio y del desarrollo profesional, los que lideran su propio proceso de mejora continua (Powell, 2000).

Shulman $(1998,62)$ afirma que una de las mayores virtudes del portafolios como herramienta para la evaluación del profesorado es que «cambia el centro de actividad, que deja de estar a cargo de un observador para volver a manos del practicante». 
Mediante el ejercicio de la autoevaluación que el profesor realiza durante el proceso de desarrollo del portafolios, se convierte en el principal agente de su desarrollo profesional:

El desarrollo de la competencia de los profesores para reflexionar sobre su propia enseñanza requiere considerar al profesor como un profesional autónomo y responsable, capaz de participar activamente en la evaluación de su propia función docente y del conjunto de componentes y elementos que configuran su actuación y, como consecuencia de todo ello, participar en la mejora de la calidad educativa (Fernández March, 2004, 130).

Se trata de un proceso de autoevaluación particularmente eficaz ya que el portafolios «se conecta con la realidad de la pedagogía de la disciplina» (Fernández March, 2003). El portafolios es capaz de reflejar la multidimensionalidad de los contenidos implicados en el proceso de enseñanza-aprendizaje, es una evaluación «multifacética y retrata la naturaleza de la enseñanza» (Klenowski, 2000, 221).

\section{SISTEMAS DE APOYO Y SEGUIMIENTO: TUTORÍA O MENTORAZGO}

El portafolios docente puede elaborarlo el propio profesor sin intervención de ninguna otra persona en el proceso de desarrollo. Sin embargo, cuando el portafolios pretende ser un instrumento de ayuda para la toma de decisiones y la mejora de la docencia del profesor, parece preferible contar con el apoyo de una persona que confronte y corrobore las evidencias introducidas en él (Freidus, 1998; Lyons, 1998; Klenowski, 2004; Seldin, 2004; Ginés, Davis y otros, 2005; Tigelaar, Dolmans, de Grave, Wolfhagen y van der Vleuten, 2006).

La docencia se considera en muchas ocasiones una profesión aislada, ya que a menudo el docente trabaja solo, sin la colaboración de otros profesores. Por ello, disponer de la ayuda de un mentor para realizar el portafolios docente es una buena oportunidad para establecer vínculos entre compañeros y fomentar la colaboración (Ginés, Davis y otros, 2005). Seldin (2004) argumenta que el desarrollo del portafolios conviene que implique cierta interacción y mentorazgo, al 
igual que una tesis doctoral refleja tanto los esfuerzos del doctorando como los consejos del director del trabajo.

Las funciones del tutor son las de prestar apoyo, ayudar a identificar tanto los puntos fuertes como las posibles necesidades, fomentar la reflexión, proporcionar retroalimentación al proceso y ayudar a establecer planes de mejora $\mathrm{u}$ objetivos a corto y medio plazo. Según Lyons $(1998,144)$ la conversación en torno al portafolios puede resultar para el profesor en una

[...] oportunidad para reconsiderar sus experiencias, para ir más allá de las entradas de su portafolios y poder comprender y establecer conexiones entre su práctica docente, el aprendizaje de sus alumnos y su crecimiento y desarrollo como profesional reflexivo.

Según Seldin $(2004,5)$, las personas que podrían desempeñar la función de mentor podrían ser el director del departamento, un colega, o un especialista en formación o en desarrollo profesional del profesorado. Sin embargo, advierte que sea quien sea el que desempeñe dicho papel, conviene que tenga conocimientos adecuados sobre los procedimientos e instrumentos efectivos para documentar o evidenciar una docencia de calidad. Sólo de esta manera el mentor podrá ayudar al profesor proporcionándole sugerencias y recursos y apoyándole de modo constante durante la preparación del portafolios.

Por otra parte, afirma que al haber diferencias entre los diversos miembros del claustro y los heterogéneos contextos institucionales, no existe una única manera de organizar dicha colaboración, aunque anota algunos procedimientos que se han mostrado útiles (Seldin, 2004, 6):

- Un profesor mayor y más experimentado que trabaja directamente con un colega más joven de la misma disciplina o de otra.

- Un grupo experto de profesores de la propia institución que, a cambio de una reducción de la carga docente, mentoriza a varios profesores mientras preparan sus portafolios.

- Un consultor o especialista externo, de la propia disciplina o de otra. 
En cualquier caso, el mentor nunca debe olvidar que el portafolios es del profesor que lo está preparando. Por ello, aunque se realiza de manera cooperativa y pueda aportar sugerencias o recomendaciones, las decisiones -sobre lo que se desea incluir, la finalidad, el estilo, etcétera-, en último término, las determina el propio profesor. «El rol del mentor es de guía, no de director» (Seldin, 2004, 6). Son los profesores que están desarrollando el portafolios quienes deciden por ellos mismos iniciar un proceso de desarrollo profesional y son ellos quienes asumen la responsabilidad de su propio aprendizaje que, con la ayuda del portafolios, les permite ir adquiriendo ciertos hábitos de reflexión. Por ello, no debe fomentarse la dependencia del tutor o de otra persona más experimentada (Klenowski, 2004, 132).

Por otra parte, no sólo la figura de un mentor puede resultar beneficiosa, sino que todos los sistemas de apoyo o seguimiento durante el proceso de desarrollo de portafolios que se le puedan brindar a los profesores, les resultarán de gran utilidad. Una de las opciones es realizar regularmente reuniones o foros con grupos de profesores que estén desarrollando sus portafolios. De esta manera, se realiza una tutoría entre pares que se reúnen para analizar y comentar su progreso. Por ello se puede afirmar que

[...] pueden ejercer la tutoría del portafolio profesores o pares, y además está la autotutoría a que la estructura misma del portafolio da lugar (Freidus, 1998, 84).

\section{CONCLUSIONES Y REFLEXIONES}

El portafolios docente puede ser un instrumento efectivo en el ámbito universitario que alberga una doble virtualidad: recurso formativo e instrumento de evaluación. Su aprovechamiento en el ámbito nacional se encuentra en sus inicios, por ello conocer las diferencias de los diversos enfoques nos dispone a desarrollar prácticas coherentes, sin limitar las posibilidades o beneficios de un determinado punto de vista. 
En el ámbito universitario existe la necesidad de valorar de manera adecuada la dimensión docente en el desempeño profesional del profesorado y equipararla de alguna manera a la importancia ya reconocida de su labor investigadora. Es necesario que el esfuerzo, la dedicación y la formación de los profesores en su labor educativa se vea recompensada e incentivada, como lo son sus publicaciones. Estamos en un ambiente en el que parece que los asuntos relacionados con la garantía de la calidad de las universidades y la evaluación de su profesorado cobran gran relevancia. Comienza a darse más peso que en otros momentos a la dimensión docente, así lo muestra, por ejemplo, el programa DOCENTIA que ha sido presentado por la Agencia Nacional de Evaluación de la Calidad y Acreditación (ANECA) 9 Conviene por ello buscar alternativas que permitan valorar los méritos docentes. En este contexto, reconocemos la posibilidad de concebir los portafolios como una colección ordenada y estructurada de evidencias que demuestran la competencia y el desarrollo del profesor universitario como docente y sugerir su utilidad en procesos de habilitación o futuras acreditaciones.

El portafolios docente es, además de un recurso de evaluación, un efectivo medio para la formación del profesorado universitario. La distinción entre una y otra modalidad (evaluativa o formativa) se establece desde el plano teórico ya que, en muchas ocasiones, esta diferencia no es operativa en la realidad. Incluso en los casos en que el portafolios se utiliza con fines evaluativos, posee una función formativa, aunque ésta no haya sido perseguida.

Aunque se trata de un organismo español, al ser la autora española, lo mismo puede decirse de la situación mexicana. «La Agencia Nacional de Evaluación de la Calidad y Acreditación (ANECA) es una fundación estatal que tiene como objetivo contribuir a la mejora de la calidad del sistema de educación superior mediante la evaluación, certificación y acreditación de enseñanzas, profesorado e instituciones.

»Este objetivo está alineado con la finalidad de construir un Espacio Europeo de Educación Superior (EEES) en el año 2010. ANECA lleva a cabo su actividad (evaluación, certificación y acreditación) a través de diferentes programas». En: [http:/ / www.aneca.es/que-es-aneca/ presentación.aspx]. Nota del editor. 
Estamos en un momento adecuado para repensar la docencia universitaria, para realizar cambios y proponer proyectos que permitan conocer, apreciar y mejorar la docencia que se desarrolla en nuestras instituciones. Podemos pensar en el potencial formativo del empleo de portafolios docentes, capaces de fomentar la práctica reflexiva en los profesores, como instrumento eficaz para proporcionar soporte a la formación del profesorado universitario.

\section{BIBLIOGRAFÍA}

AGRA, M.J., GEWERC, A. y MONTERO, L., «El portafolios como herramienta de análisis en experiencias de formación on line y presenciales», comunicación presentada al II Congreso Europeo de Tecnologías de la Información en la Educación y la Ciudadanía: Una Visión Crítica, Barcelona, 2002, en: [http://web.udg.es/tiec/ orals/c45.pdf]. Consultado: 9-XII-2008.

BARRETT, H., Electronic Teaching Portfolios: Multimedia Skills + Portfolio Development $=$ Powerful Professional Development, in: [http:/ / electronicportfolios.com/portfolios/3107Barrett.pdf]. 9-XII-2008.

BARRETT, H.C. (2005a). My «On line Portfolio Adventure» (Versions of my on line portfolios developed using different systems or on line publishing tools), in: [http:/ / electronicportfolios.org/ myportfolio/ versions.htlm]. 9-XII-2008.

BARRETT, H C. (2005b). Create on line portfolios using common tools and open source softwar, in: [http: / / center.uoregon.edu/ISTE/ uploads / NECC2005/KEY_6807039/Barrett_NECC05workshop. pdf]. 9-XII-2008.

BARRETT, H.C. (2005c). Directions for ePortfolio Research, in: [http:/ / electronicportfolios.com/research.html]. 9-XII-2008. 
BARRETT, H. \& CARNEY, J. (2003), Electronic Portfolios: Decisions, and Dilemas, in: [http: / / electronicportfolios.com/ portfolios/ AAHE2003.pdf]. 9-XII-2008.

BIRD, T., «The Schoolteacher's Portfolio: An Essay on Possibilities», in MILLMAN, J. \& DARLING-HAMMOND, L. (eds.), The New Handbook of Teacher Evaluation. Assessing Elementary and Secondary School Teachers, SAGE Publications, California, 1990.

CAMPBELL, D.M.; CIGNETTI, P.B.; MELENYZER, B.J.; NETTLES, D.H. \& WYMAN, R.M., How to Develop a Professional Portfolio: a Manual for Teachers, 3rd ed., Pearson Education, Boston, 2004.

CANO, E., El portafolios del profesorado universitario. Un instrumento para la evaluación y para el desarrollo profesional, Octaedro, Barcelona, 2005.

CERBIN, W., «The Course Portfolio as a Tool for Continuous Improvement of Teaching and Learning», Journal on Excellence in College Teaching, 5:1, 1994, p. 95-105.

CLAY, A.S.; PETRUSA, E.; HARKER, M. \& ANDOLSEK, K., «Development of a web-based, specialty specific portfolio», Medical Teacher, 29:4, 2007, p. 311-316.

COFFEY, A., «The Clinical Learning Portfolio: a Practice Development Experience in Gerontological Nursing», Journal of Older People Nursing in association with Journal of Clinical Nursing, 14:8b, 2005, p. 75-83.

COLE, D.J., Portfolios Across the Curriculum and Beyond, Corwin Press, California, 1995.

CONRAD, L. \& BOWIE, C., «The Impact of Context on Readings of Teaching Portfolios», International Journal for Academic Development, 11:1, 2006, p. 31-42.

CONSTANTINO, P.M. \& DE LORENZO, M.N., Developing a Professional Teaching Portfolio: a Guide for Success, Allyn and 
Bacon, Boston.

CRISPÍN, M.L. y CAUDILLO, L., El uso del portafolios como herramienta para mejorar la calidad de la docencia, 1998, en:

[http: / / www.comunidades.ipn.mx/descargas / Modelo/portafolio.pdf]. 9-XII-2008.

DANIELSON, C. \& ABRUTYN, L., An introduction to using portfolios in the classroom, Association for Supervision and Curriculum Development, Alexandria, 1997.

DE MIGUEL, M., «La evaluación del profesorado universitario. Criterios y propuestas para mejorar la función docente», Revista de Educación, 315, 1998, p. 67-83.

DOEL, M., Learning, Practice and Assessment: Signposting the Portfolio, Philadelphia: Jessica Kingsley, London, 2002.

DUQUE, G.; FINKELSTEIN, A.; ROBERTS, A.; TABATABAI, D.; GOLD, S. \& WINER, L., Learning while Evaluating: the Use of an Electronic Evaluation Portfolio in a Geriatric Medicine Clerkship, 2006, in: [http://www.biomedcentral.com/14726920/6/4]. 9-XII-2008.

EDGERTON, R., HUTCHINGS, P. \& QUINLAN, K., The teaching portfolio: capturing the scholarship in teaching, American Association for Higher Education, Washington, 1991.

FEIXAS, M. y VALERO, M., El portafolios y el SEEQ como herramientas para el desarrollo profesional, 2003, en: [http: / / www.udg.edu /ice/FUniversit/Portafolios.pdf]. 9-XII-2008.

FERNÁNDEZ MARCH, A., «El portafolio docente como estrategia formativa y de desarrollo profesional», Educar, 33, 2004, p. 127-142.

FREIDUS, H., «El papel del tutor en la elaboración del portafolio», 1998, p. 80-101, en LYONS, N. (ed.), With Portfolio in Hand. Validating the New Teacher Professionalism, Teachers College Press, Columbia. Ed. esp.: El uso de portafolios. Propuestas para 
un nuevo profesionalismo docente, Amorrortu, Buenos Aires, 2003.

GINES, M.; DAVIS, S. \& BORDERA, C., Portfoli Docent, 2005, in: [https: / / sarasate.upc.es/upc/ice/bbdd/profi.nsf/PortfoliDocentD et]. 9-XII-2008.

HOLMBOE, E.; RODAK, W.; MILLS, G.; McFARLANE, M. \& SCHULTZ, H., "Outcomes-based Evaluation in Resident Education: Creating Systems and Structured Portfolios», The American Journal of Medicine, 119:8, 2006, p. 708-714.

HUTCHINGS, P. (ed.), The Course Portfolio: How Faculty Can Examine Their Teaching to Advance Practice and Improve Student Learning, American Association for Higher Education, Washington, 1998.

KINGORE, B.W., Portfolios: enriching and assessing all students, identifying the gifted grades K-6, Leadership Publishers, Des Moines, Iowa, 1993.

KLENOWSKI, V., «Portfolios: Promoting Teaching. Assessment in Education: Principles», Policy \& Practice, 7:2, 2000, p. 215- 236.

KLENOWSKI, V., Developing Portfolios for Learning and Assessment: Processes and Principles, RoutledgeFalmer, London/New York, 2002.

KLENOWSKI, V.; ASKEW, S. \& CARNELL, E., «Portfolios for learning, assessment and professional development in higher education», Assessment \& Evaluation in Higher Education, 31:3, 2006, p. 267-286.

KNAPPER, C. \& WILCOX, S., Preparing a Teaching Dossier, Instructional Development Centre, Queen's University, Canada, 1998. Ed. esp.: El portafolios docente, Red Estatal de Docencia Universitaria, Madrid, 2003.

LABOSKEY, V.K., «Teaching to Teach with Purpose and Passion: Pedagogy for Reflective Practice», 1997, p. 150-163, in 
LOUGHRAN, J. \& RUSSELL, T. (eds.), Teaching about Teaching: Purpose, Passion and Pedagogy in Teacher Education, Falmer Press, London.

LORENZO, G. \& ITTELSON, J., An Overview of E-Portfolios, 2005, in: [http: / / www.educause.edu/ir/library/pdf/ELI3001.pdf]. 9-XII-2008.

LYONS, L., With Portfolio in Hand. Validating the New Teacher Professionalism, Teachers College Press, Columbia, 1998.

MARTÍNEZ MUT, B.; FERNÁNDEZ MARCH, A.; GROS, B. y ROMAÑA, T., El cambio de cultura docente en la universidad y el Espacio Europeo de Educación superior, 2005, en: [http:/ / www.ice.upv.es/ site05/Ponencia2.pdf]. 9-XII-2008.

MARTIN-KNIEP, G.O., Capturing the Wisdom of Practice, Association for Supervision \& Curriculum Development, Alexandria, 1999. Ed. esp.: Portfolios del desempeño de maestros, profesores y directivos, Paidós, Buenos Aires, 2001.

MULLEN, L.; BRITTEN, J. \& McFADDEN, J., Digital Portfolios in Teacher Education, JIST Works, Indianapolis, 2005.

MURRAY, J.P., Successful Faculty Development and Evaluation: the Complete Teaching Portofolio, ERIC Clearinghouse on Higher Education, Washington, DC, 1997.

POWELL, L.A., "Realising the Value of Self-assessment: the Influence of the Business Excellence Model on Teacher Professionalism», European Journal of Teacher Education, 23:1, 2000, p. 37-48.

RICO VERCHER, M. y RICO PÉREZ, C., El portafolio discente, Editorial Marfil, Alcoy, 2004.

ROLHEISER-BENNETT, N.C., The Portfolio Organizer Succeeding with Portfolios in Your Classroom, Association for 
Supervision \& Curriculum Development, Alexandria, 2000.

SANTOS, M.A., La evaluación: un proceso de diálogo, comprensión y mejora, Aljibe, $2^{a}$ ed., Málaga, 1995.

SELDIN, P., The Teaching Portfolio: a Practical Guide to Improved Performance and Promotion/Tenure Decisions, Anker Press, Bolton, 1991.

SELDIN, P., Successful Use of Teaching Portfolios, Anker Press, Bolton, 1993.

SELDIN, P., The Teaching Portfolio. A Practical Guide to Improved Performance and Promotion/Tenure Decisions, Third ed., Anker Publishing Company, Bolton, 2004.

SHULMAN, L., «Portafolios del docente: una actividad teórica», p. 44-62, en LYONS, N. (ed.), With Portfolio in Hand. Validating the New Teacher Professionalism, Teachers College Press, Columbia, 1998. Ed. esp.: El uso de portafolios. Propuestas para un nuevo profesionalismo docente, Amorrortu editores, Buenos Aires, 2003.

SMITH, K. \& TILLEMA, H., «Long-term Influences of Portfolios on Professional Development», Scandinavian Journal of Educational Research, 45:2, 2001, p. 183-203.

SMITH, K. \& TILLEMA, H., "Clarifying Different Types of Portfolio Use», Assessment \& Evaluation in Higher Education, 28:6, 2003, p. 625-648.

STANLEY, C., «The Faculty Development Portfolio: A Framework for Documenting the Professional Development of Faculty Developers», Innovative Higher Education, 26:1, 2001, p. 23-36.

THOMAS, D.A., «Interdisciplinary Effectiveness and The Learning Portfolio: Developing Required in the 21st Century Workpace», in ZUBIZARRETA, J. (ed.) The Learning Portfolio: Reflective Practice for Improving Student Learning, Anker Publishing Company, Bolton, 2004, p. 146-152.

TIGELAAR, D.; DOLMANS, D.; DE GRAVE, W.; WOLFHAGEN, I. \& VAN DER VLEUTEN, C., «Participants' opinions on the usefulness of 
a teaching portfolio», Medical Education, 40, 2006, p. 371-378.

TILLEMA, H., «Design and Validity of a Portfolio Instrument for Professional Training», Studies In Educational Evaluation, 24:3, 1998, p. 263-278.

TUCKER, P.D., STRONGE, J.H. \& GAREIS, C.R., Handbook on Teacher Portfolios for Evaluation and Professional Development, Eye on Education, Larchmont, NY, 2002.

VERA, J., «La evaluación de los profesores: dificultades, aceptaciones y rechazos», comunicación presentada al VIII Congreso Nacional de Teoría de la Educación, Huelva, 2001.

WADE, R.C. \& YARBROUGH, D.B., «Portfolios: A tool for Reflective Thinking in Teacher Education?», Teaching and Teacher Education, 12:1, 1996, p.63-79.

WHITTIER, D. \& LARA, S., «US vs. European E-folio Design: Investigating a Dichotomy», Society of Information Technology and Teacher Education SITE. 16th International Conference, Phoenix, Arizona, 2005.

WOOD, B. \& MAY, W., «Academic Recognition of Educational Scholarship», Acad Radiol, 13, 2006, p.254-257.

WRAY, S., «Teaching portfolios, community, and pre-service teachers' professional development», Teaching and Teacher Education, 23: 7, 2007, p. 1139-1152.

ZUBIZARRETA, J., How Teaching Portfolios Improve Course Instruction, The Departament Chair, 5(4), 1995, p. 15-16.

ZUBIZARRETA, J. (ed.), The Learning Portfolio: Reflective Practice for Improving Student Learning, Anker Publishing Company, Bolton, 2004. 